\title{
Análise espaço-temporal da mortalidade por suicídio no Rio Grande do Norte, Brasil, no período de 2000 a 2015
}

\author{
Space-time analysis of mortality by suicide in the State of Rio \\ Grande do Norte, Brazil, in the period from 2000 to 2015
}

Emelynne Gabrielly de Oliveira Santos (https://orcid.org/0000-0002-3890-4879) ${ }^{1}$

Isabelle Ribeiro Barbosa (https://orcid.org/0000-0002-1385-2849) ${ }^{1}$

Ana Kalliny Sousa Severo (https://orcid.org/0000-0002-9548-6394) ${ }^{1}$

${ }^{1}$ Faculdade de Ciências da Saúde do Trairi, Universidade Federal do Rio Grande do Norte. Avenida Rio Branco, Centro. 59200000 Santa Cruz RN Brasil. emeoliveirasantos@ hotmail.com

\begin{abstract}
The objective of this study was to analyze the space-time distribution of mortality by suicide in the State of Rio Grande do Norte (RN) between 2000 and 2015. This is a mixed ecological study that evaluated the deaths due to suicide registered in RN. Spatial analysis was performed using crude and standardized rates. Univariate analysis was conducted using the Moran Global and Local Index, and spatial bivariate analysis was assessed using the cross-referencing of sociodemographic variables and mortality rates. By applying Joinpoint Regression, it was possible to verify the suicide mortality trend. There were 2,266 suicide deaths from 2000 to 2015, and spatial analysis showed poor spatial autocorrelation $(I<0.3)$ for the Standardized Mortality Rates for both sexes, with formation of spatial clusters in the Serido Region. The bivariate analysis showed the formation of clusters in the Serido Region with HDI and Aging variables. In addition, there was a trend of increased male mortality up to 2003 $(A P C=15.3 \%, C I=9.2-21.8)$, followed by a period of stability.
\end{abstract}

Key words Suicide, Mortality, Social determinants of health, Spatial analysis, Time series studies

Resumo O objetivo deste estudo foi analisar a distribuição espaço-temporal da mortalidade por suicídio no Rio Grande do Norte (RN) no periodo de 2000 a 2015. Trata-se de um estudo ecológico misto que avaliou os óbitos decorrentes de suicídio registrados no RN. A análise espacial foi realizada utilizando as taxas brutas e padronizadas. A análise univariada foi realizada através do Indice de Moran Global e Local, e a análise bivariada espacial por meio do cruzamento entre as variáveis sociodemográficas e as taxas de mortalidade. Através da Regressão Joinpoint foi possivivel verificar a tendência de mortalidade por suicídio. Foram registrados 2.266 óbitos por suicídio de 2000 a 2015, e a análise espacial mostrou fraca autocorrelação espacial $(I<0,3)$ para as Taxas de Mortalidade Padronizadas para ambos os sexos, com formação de aglomerados espaciais na Região do Seridó. A análise bivariada mostrou formação de clusters na Região do Seridó com as variáveis IDH e Envelhecimento. Além disso, foi observada uma tendência de aumento da mortalidade no sexo masculino até $o$ ano de 2003 ( $A P C=15,3 \%$; IC = 9,2-21,8), seguido de um período de estabilidade.

Palavras-chave Suicídio, Mortalidade, Determinantes sociais da saúde, Análise espacial, Estudos de Séries Temporais 


\section{Introdução}

Considerado um dos tipos de mortes violentas mais incidentes no mundo, o suicídio configura-se como um ato deliberado, executado pelo próprio indivíduo, cujo intento é a morte de forma consciente e intencional. Além disso, constitui-se como um fenômeno complexo, de caráter violento, ou seja, um evento intencional que corresponde às causas externas de mortalidade representado na Classificação Internacional de Doenças (CID); agressivo, e de forte impacto na sociedade ${ }^{1}$.

Com a estimativa de uma morte a cada 40 segundos, a mortalidade por suicídio varia de acordo com o tempo e espaço. Para o ano de 2020, estima-se um aumento de $50 \%$ na incidência anual de mortes por suicídio em todo o mundo. No Brasil, entre os anos 2011 e 2015, foram registradas 55.649 óbitos, com taxa geral de 5,5/100 mil habitantes, com destaque para altas taxas de na população economicamente ativa $-7,9 / 100 \mathrm{mil}$ habitantes em indivíduos entre 40-49 anos, refletindo diretamente em indicadores que retratam as mortes ocorridas precocemente, como os Anos Potenciais de Vida Perdidos (APVP) ${ }^{1-3}$.

As predições de óbitos por suicídio estão fortemente associadas ao processo de transição demográfica e epidemiológica observada no Brasil ${ }^{4}$. Isso pode ser explicado pelo aumento na expectativa de vida, na mortalidade por causas externas e pela elevada incidência de transtornos mentais na população - depressão, ansiedade, esquizofrenia; e uso de substâncias psicoativas, que constituem perfil dos fatores de risco associados ao suicídio ${ }^{5}$.

O panorama da mortalidade por suicídio no Brasil é preocupante, principalmente em regiões em que se observa uma elevação na taxa de mortalidade no decorrer dos anos. No Nordeste, a taxa de mortalidade variou de 2,3 para 4,49/100mil habitantes entre os anos 2000 e 2015. Entretanto, as semelhanças e disparidades entre as diversas regiões suscitam a necessidade de politicas públicas que possam atender as peculiaridades de cada região ${ }^{6}$.

No estado do Rio Grande do Norte (RN), verificou-se um aumento de $83,5 \%$ na mortalidade por causas externas entre os anos 2000 e 2015. No mesmo período, a taxa de mortalidade por lesões autoprovocadas intencionalmente aumentou $46 \%$ no total da população, com variação de 2,9 a 4,5/100 mil habitantes, respectivamente. Um estudo mostrou, ainda, que o município de Caicó, localizado na Região do Seridó, interior do estado do RN, apresentou taxa de 15,8/100 mil habitantes entre os anos 2005 e 2007, ocupando o $3^{\circ}$ lugar entre as 20 cidades brasileiras, com pelo menos 50.000 habitantes, com maiores coeficientes de suicídio ${ }^{6,7}$.

É importante ressaltar que estudos sobre a mortalidade por suicídio no Rio Grande do Norte ainda são escassos. Quando existem, estão restritos a populações mais específicas e se tornam mais raros aqueles que utilizam metodologias de tendências e análises espaciais. Sem o entendimento de como o fenômeno se apresenta no espaço, as ações de saúde tornam-se ineficientes, uma vez que não há um direcionamento para grupos e regiões vulneráveis.

Considerando o exposto, esse estudo tem como objetivo analisar a tendência temporal e a distribuição espacial da mortalidade por suicídio no RN e entender sua relação com o contexto socioeconômico em que a população está inserida, buscando ir além dos dados pontuais de taxas ao se obter informações que possam constatar disparidades socioespaciais. Esses dados serão úteis para o planejamento, consolidação e avaliação de políticas de saúde; e fornecerão subsídios para ações de reorganização das redes de atenção em saúde, sobretudo no que se refere ao fortalecimento das ações no campo da saúde mental, reduzindo o impacto futuro da mortalidade por essa causa.

\section{Métodos}

Trata-se de um estudo ecológico misto que avaliou os óbitos decorrentes de suicídio registrados no Rio Grande do Norte. Para Medronho ${ }^{8}$, esse tipo de desenho contempla uma associação entre tempo e espaço, ou seja, considera as diversas alterações observadas em múltiplos grupos ao longo do tempo e em diferentes locais.

A escolha do período estudado decorreu da possibilidade de se discutir a temporalidade com maior robustez ${ }^{9}$. Além disso, foi observado que a partir da década de 2000 o Sistema de Informação sobre Mortalidade vem ganhando maior qualidade, principalmente no Norte e Nordeste, o que assegura a maior qualidade dos dados ${ }^{10,11}$.

A variável dependente foi a Taxa de Mortalidade Padronizada (TMP) de suicídio. Para o cálculo da TMP, foi utilizada a média dos óbitos decorrentes de lesões autoprovocadas intencionalmente categorizadas a partir da Classificação Estatística Internacional de Doenças e Problemas Relacionados à Saúde - 10a Revisão (X60-84), 
ocorridas no período de $1^{\circ}$ de janeiro de 2010 a 31 de dezembro de 2015. Os óbitos foram coletados do Sistema de Informação sobre Mortalidade (SIM) do Departamento de Informática do Sistema Único de Saúde (Datasus) ${ }^{7}$. Os dados de população por município e por idade foram obtidos das informações do Censo 2010 e das projeções intercensitárias, no sítio do Instituto Brasileiro de Geografia e Estatística. As taxas brutas foram padronizadas pelo método direto, considerando a população padrão brasileira, e expressas por 100 mil habitantes por ano ${ }^{12-14}$.

As variáveis independentes foram analisadas em dois níveis: contextuais e individuais. As primeiras representam os indicadores socioeconômicos e são referentes aos censos 2000 e 2010, sendo obtidas através do Atlas de Desenvolvimento Humano no Brasil do Programa das $\mathrm{Na}-$ ções Unidas para o Desenvolvimento (PNUD): Índice de GINI, IDH-M, Taxa de Envelhecimento, Taxa de Analfabetismo de 25 anos ou mais, Percentual de vulneráveis à pobreza, Taxa de desocupação de 18 anos ou mais e População rural. As variáveis individuais consideradas para esse estudo foram retiradas do Sistema de Informação sobre Mortalidade: Sexo e Faixa etária ${ }^{7,14}$.

Para análise de dependência espacial, utilizou-se o índice de Moran Global que estima a autocorrelação espacial, e pode variar entre - 1 e +1 ; além disso, pode fornecer a significância estatística do mesmo (valor de p). Posteriormente, foi avaliada a presença de clusters através do Moran local (Local Indicators of Spatial Association LISA). Dessa forma, foi construído o MoranMap relativo às taxas de Mortalidade por suicídio, apresentando apenas àqueles clusters com valor de $\mathrm{p}<0,05$. Para a validação do Índice de Moran Global, foi utilizado o teste de permutação aleatória, com 99 permutações. O software Terraview 4.2.2 foi utilizado para a produção dos mapas temáticos em quadriênios e para o cálculo do Índice de Moran Global e Local ${ }^{15}$.

A análise bivariada LISA para avaliação da correlação espacial entre a variável dependente e as variáveis independentes foi realizada utilizando-se o software GeoDa 1.6.61 (Spatial Analysis Laboratory, University of Illinois, Urbana Champaign, Estados Unidos). Para tanto, foram construídos os mapas temáticos com cada par de variáveis, sendo verificado o padrão de dependência espacial e a sua significância estatística.

Para analisar a tendência de mortalidade utilizou-se a análise de regressão Joinpoint, através do software Joinpoint Regression Program (National Cancer Institute, Bethesda, Maryland,U-
SA), Versão 4.5.0. Esse modelo é aplicado para calcular a mudança percentual anual da taxa de mortalidade (APC). Para este estudo, a variável dependente foi do tipo taxa ajustada por idade (100.000 habitantes), e a variável independente escolhida foi o ano.

Essa pesquisa utilizou dados secundários disponíveis em sites oficiais do Ministério da Saúde do Brasil, sem identificação de sujeitos, sendo dispensada de apreciação em comitê de ética em pesquisa, em conformidade com a Resolução 466/2012 do Conselho Nacional de Saúde.

\section{Resultados}

De 2000 a 2015, ocorreram 2.266 óbitos por suicídio no Rio Grande do Norte, com $81,1 \%$ dos óbitos afetando o sexo masculino e $18,9 \%$ o sexo feminino. A taxa de mortalidade padronizada (TMP) à população mundial para os homens no RN variou de 4,8 óbitos/100.000 habitantes, no ano de 2000, a 7,7 óbitos/100.000 habitantes, no ano de 2015 . Para as mulheres, essa taxa variou de 1,3 óbitos/100.000 habitantes, no ano de 2000, a 1,6 óbitos/100.000 habitantes no ano de 2015 .

A análise espacial para as taxas de mortalidade brutas e padronizadas, para o sexo feminino, mostrou que não há autocorrelação espacial para a TMP por suicídio no Rio Grande do Norte (Figura 1). O valor de Moran Global obtido aponta para uma fraca autocorrelação espacial, com significância estatística para 5 quadriênios. Para o sexo masculino, também foi observado esse mesmo padrão, embora o valor de Moran Global obtido tenha se apresentado mais elevado quando comparado ao sexo feminino para maioria dos quadriênios (Figura 2).-

$\mathrm{Na}$ análise do padrão espacial das taxas de mortalidade através do MoranMap, foi verificada a presença de pequenos aglomerados de alta taxa de mortalidade na região do Seridó, sobretudo para o sexo masculino, conforme verifica-se na Figura 3.

Já na análise bivariada, através do Índice de Moran Local Bivariado (LISA), foi observada uma fraca autocorrelação espacial entre as variáveis socioeconômicas do estudo e a TMP por suicídio no RN no primeiro e último quadriênio (Figuras 4 e 5, respectivamente). Nos dois quadriênios, por sua vez, observou-se a formação de clusters na região do Seridó do RN de elevada TMP com elevados valores das variáveis IDH e taxa de envelhecimento - região geográfica e cultural cujo território abrange as microáreas oci- 


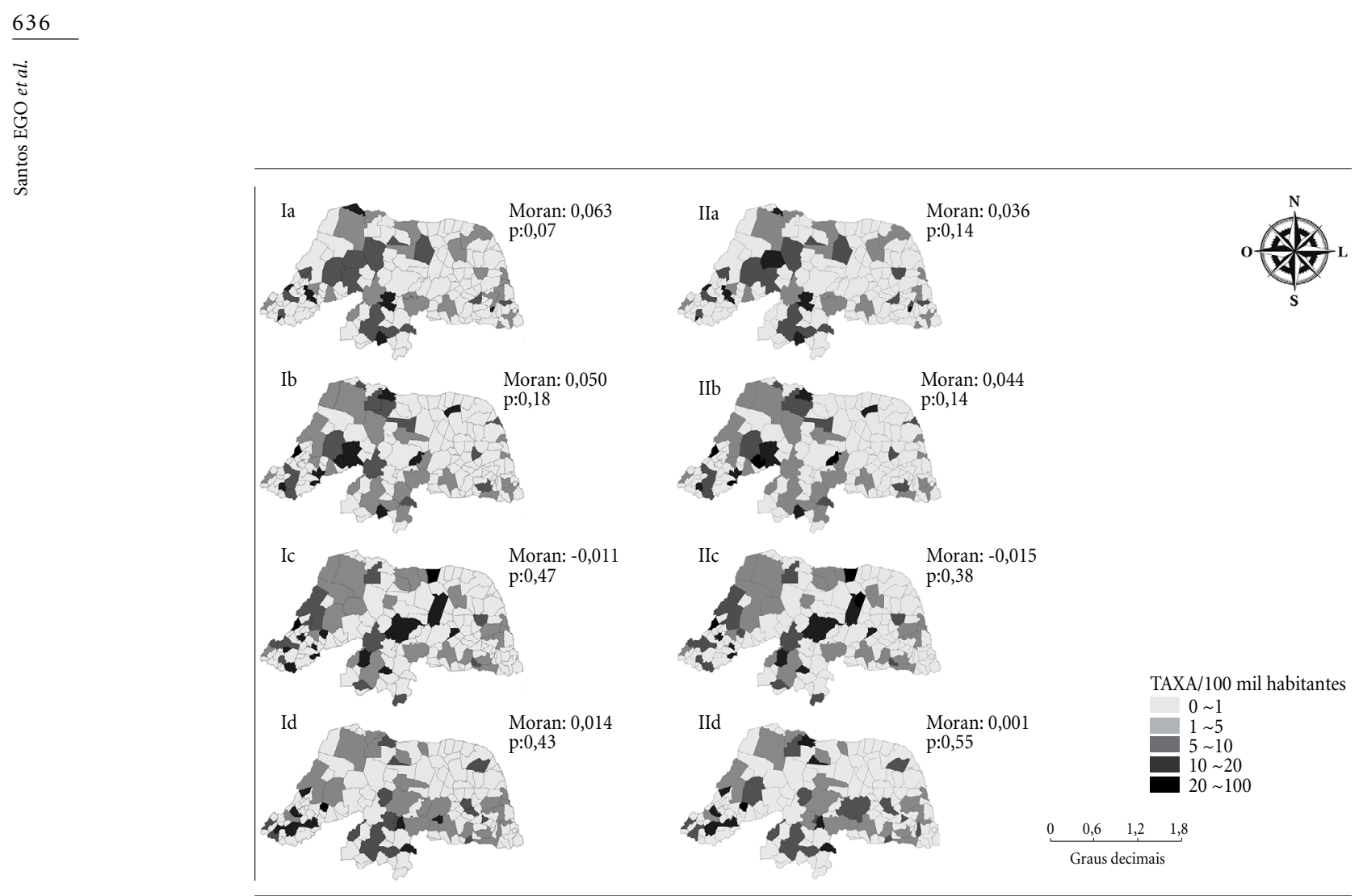

Figura 1. Distribuição espacial das taxas de mortalidade bruta (TMB) e padronizada (TMP) por suicídio no Rio Grande do Norte para o sexo feminino por quadriênio (2000 a 2015). TMB - Ia: 2000-2003, Ib: 2004-2007, Ic: 2008-2011, Id: 2012-2015; TMP - IIa: 2000-2003, IIb: 2004-2007, IIc: 2008-2011, IId: 2012-2015.

Fonte: Sistema de Informação sobre Mortalidade.

dental e oriental, divididas em 24 municípios $^{16}$. Todavia, no primeiro quadriênio (Figura 4), houve formação de clusters de altos índices de TMP para a variável "População rural” no Oeste potiguar.

A análise de tendência pelo Joinpoint verificou estabilidade para a mortalidade geral no RN (APC $=1,1 \%$ ao ano), não sendo observada significância estatística $(\mathrm{p}=0,25)$, embora a inclinação da reta, equivalente à taxa de mortalidade ajustada por idade ao longo da série histórica, seja ascendente (Gráfico 1). Entretanto, no Gráfico 2 , é possível observar que a análise de tendência no sexo feminino apresentou estabilidade ( $\mathrm{APC}=0,4 \%$ ao ano), enquanto que no sexo masculino houve uma tendência estatisticamente significativa ( $\mathrm{p}=0,03$ ) de aumento da mortalidade, com APC de 15,3\% ao ano, no período de 2000 a 2003 . (Tabela 1 ).

\section{Discussão}

O estudo da distribuição espaço-temporal da mortalidade por suicídio no Rio Grande do Nor- te permitiu observar a presença de dois padrões de correlação espacial que variam na medida em que se utilizam técnicas distintas: fraca autocorrelação para as taxas brutas, e um segundo padrão, revelando uma autocorrelação espacial para as taxas de mortalidade padronizadas que, embora fraca, apresenta-se mais autocorrelacionada e homogeneizada.

Os resultados desse estudo apontam para diferenças na distribuição da mortalidade nas regiões do RN, sendo as mais desenvolvidas as que apresentam maiores taxas. A partir desse dado, é possível inferir que o suicídio está associado às melhores condições de vida.

Para toda a série histórica estudada, as maiores magnitudes das taxas de mortalidade por suicídio, no período de 2000 a 2015, foram observadas em algumas microrregiões, como a do Seridó, mostrando, inclusive, formação de clusters alto-alto para a variável IDH. Entretanto, a literatura mostra-se escassa com estudos que abordem os possíveis determinantes que explique as elevadas taxas nessa região.

Sabe-se, porém, que o setor agropecuário apresenta-se fortemente enraizado na cultura e 


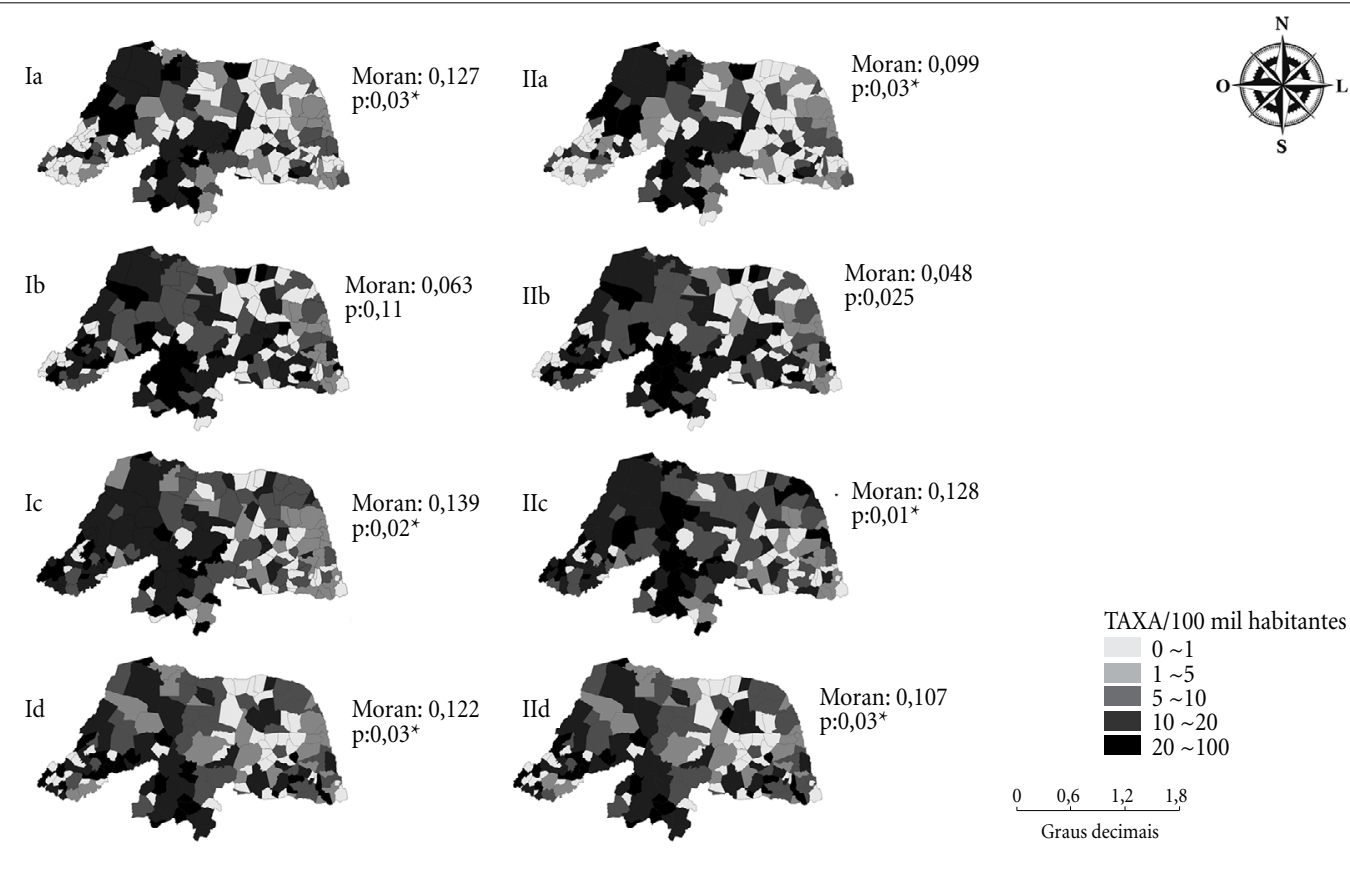

Figura 2. Distribuição espacial das taxas de mortalidade bruta (TMB) e padronizada (TMP) por suicídio no Rio Grande do Norte para o sexo masculino por quadriênio (2000 a 2015). TMB - Ia: 2000-2003, Ib: 2004-2007, Ic: 2008-2011, Id: 2012-2015; TMP - IIa: 2000-2003, IIb: 2004-2007, IIc: 2008-2011, IId: 2012-2015.

Fonte: Sistema de Informação sobre Mortalidade.

economia do interior do RN, principalmente na região do Seridó. Desse modo, buscando compreender o perfil epidemiológico do suicídio no RN, Dutra ${ }^{17}$ identificou, entre os anos 1985 e 1996, um elevado percentual de óbitos por suicídio em agricultores do interior do Estado (16,2\% em relação ao total da população do estudo).

Fraga $^{18}$, mostrou em um estudo realizado para o Brasil e Rio Grande do Sul que um dos principais fatores que afetam o número de casos de suicídio é o nível de dependência agropecuária. Percebeu-se que nos municípios com elevados números de mortes, essa característica parece ser um dos grandes determinantes, indicando uma possível associação entre as taxas de mortalidade por suicídio e a atividade agropecuária.

Por outro lado, o efeito das variáveis econômicas nas altas taxas de suicídio tem sido amplamente estudado. Nesse estudo, foram observadas elevadas taxas de mortalidade em regiões socioeconomicamente desenvolvidas. Um estudo realizado na Europa identificou que as variáveis econômicas e climáticas estão fortemente correlacionadas com as taxas de suicídio e podem explicar grande parte da variabilidade observada em todo o continente europeu, especialmente nos homens. Os resultados do estudo mostraram que o efeito climático é mais forte do que o econômico, em ambos os sexos; porém, o efeito relativo ao clima, em comparação com as variáveis econômicas, apresentou-se maior para as mulheres ${ }^{19}$.

Santos e Barbosa ${ }^{20}$, ao avaliarem a distribuição espacial da mortalidade por suicídios no Nordeste do Brasil e analisarem sua relação com indicadores sociais e econômicos, não observaram autocorrelação espacial para as taxas de mortalidade por suicídio e as variáveis socioeconômicas, uma vez que o valor de Moran Global obtido mostrouse fraco ao ser negativo e/ou próximo de zero. Esses resultados encontram-se em conformidade com os apresentados no presente estudo.

De acordo com a teoria econômica do suicídio de Hamermesh ${ }^{21}$, quanto maior a renda esperada, mais elevado será o nível de consumo e satisfação, e menor será o risco de suicídio. Em contrapartida, Durkheim ${ }^{22}$ aponta que a renda elevada favorece a taxa de suicídio uma vez que aumenta a independência pessoal e consequentemente causa degeneração familiar. Apesar dessas divergências, 


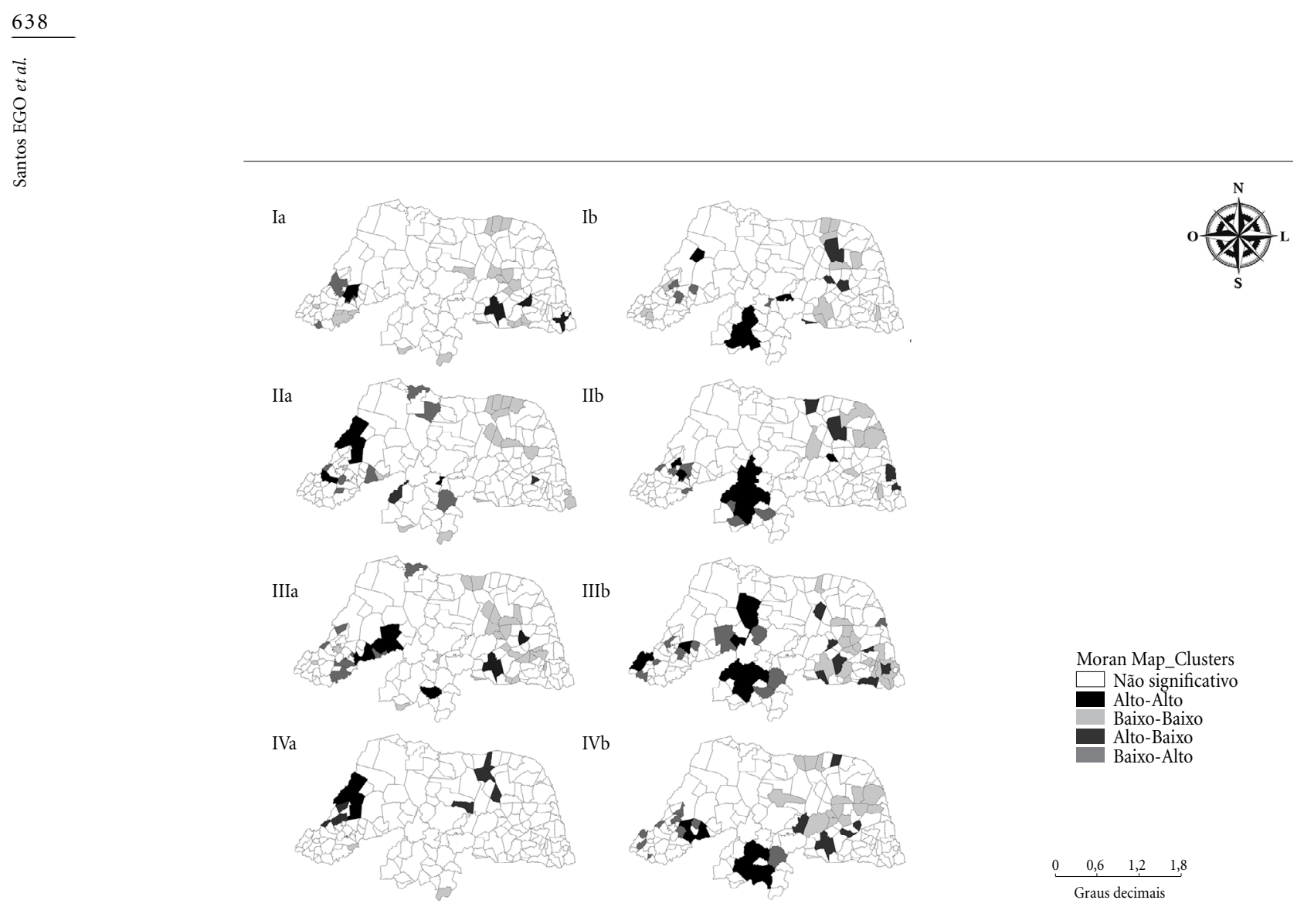

Figura 3. MoranMap da Taxa de Mortalidade Padronizada (TMP) por suicídio no RN de acordo com o sexo e por quadriênio (2000-2015). Sexo Feminino - Ia: 2000-2003, IIa: 2004-2007, IIIa: 2008-2011, IVa: 2012-2015. Sexo Masculino - Ib: 2000-2003, IIb: 2004-2007, IIIb: 2008-2011, IVb: 2012-2015.

a maioria dos estudos concorda com a hipótese de que a desigualdade de renda eleva a taxa de suicí$\mathrm{dio}^{23-25}$.

Estudos de revisão que reúnem dados em todo o mundo e refletem sobre a associação entre o suicídio e indicadores socioeconômicos mostram que altos níveis de desigualdade estão associados com baixas taxas de suicídio, enquanto elevados níveis de educação associam-se a altas taxas ${ }^{20,26,27}$.

Os resultados de um estudo realizado para microrregiões brasileiras mostraram a ausência de correlação entre a pobreza e o suicídio. Porém, constatou-se uma relação direta entre a ruralização da microrregião e taxas de suicídio $^{28}$. Para Durkheim $^{22}$, o suicídio é mais frequente em ambientes rurais e não em cidades. Esses dados corroboram com os resultados encontrados por esse estudo, em que observou-se a formação de clusters de altos índices de taxas de suicídio para a variável “população rural” em uma microrregião do RN.

Além disso, chama atenção, nesse estudo, a formação de clusters de alta taxa de suicídio e alta taxa de envelhecimento para Região do Seridó. Esse dado pode ser explicado pela mudança na transição demográfica, conceituada como a passagem de um elevado padrão de natalidade e mortalidade, para uma realidade de baixos valores dessas taxas ${ }^{4}$. Desse modo, um dos efeitos analisados nesse processo é o envelhecimento populacional, claramente observado com o aumento no número de idosos.

O Sul do Brasil concentra os maiores coeficientes de mortalidade por suicídio e, coincidentemente, de idosos ${ }^{14,23}$. As altas taxas de suicídio, nesse contexto, podem estar relacionadas ao fluxo migratório de idosos para essa região. Segundo Campos e Barbieri ${ }^{29}$, os indivíduos com melhores condições sociais, econômicas e de saúde, descolam-se após aposentadoria a fim de desfrutar dos benefícios; além daqueles que migram em busca do suporte frente às carências físicas e/ou financeiras.

Além disso, percebeu-se que a utilização de técnicas espaciais permitem estabelecer um padrão mais fidedigno de análise de distribuição espacial do suicídio. Uma dessas técnicas utiliza as taxas Bayesianas Empíricas, sendo possível observar uma distribuição mais homogênea das taxas 


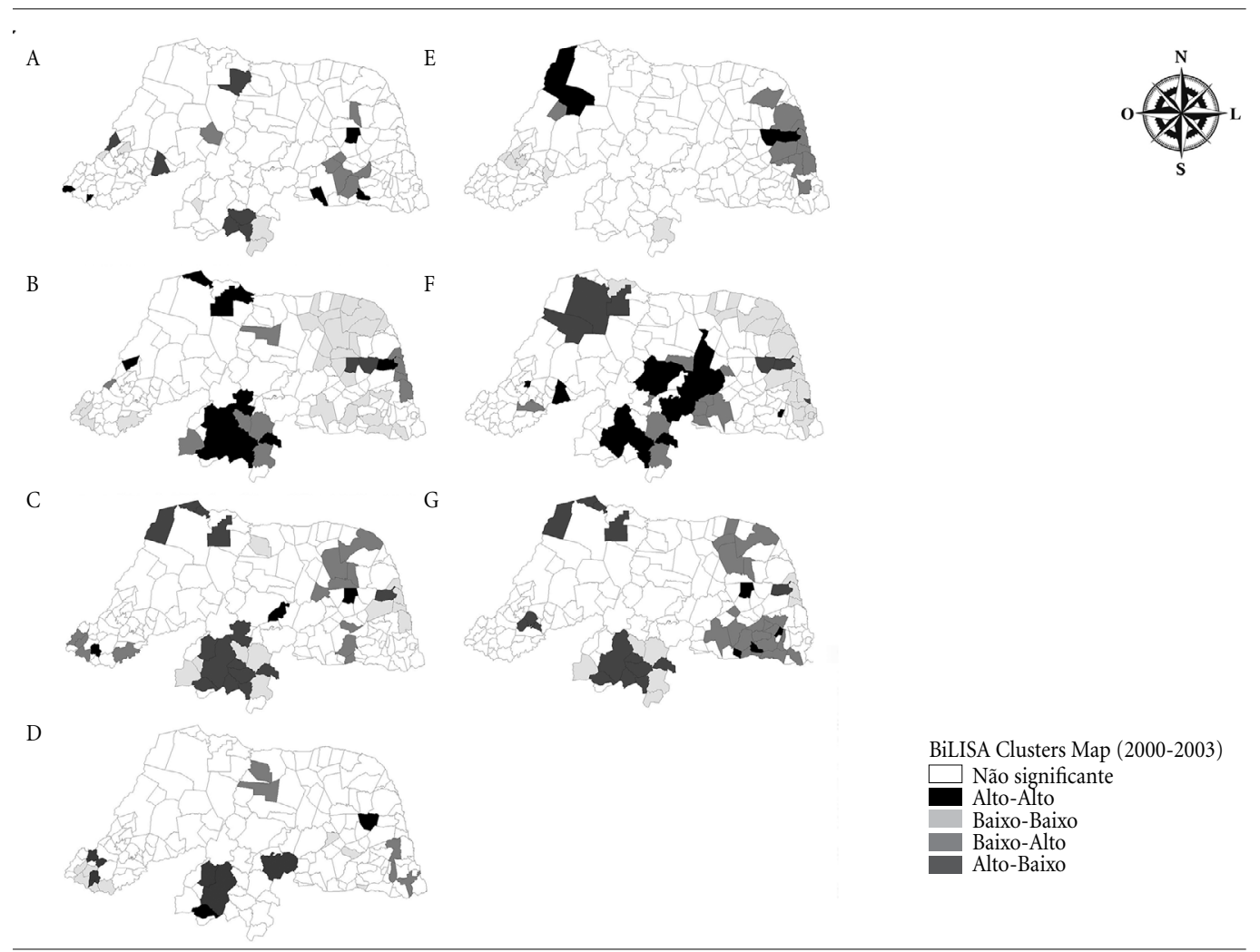

Figura 4. Moran Bivariado LISA entre as Taxas de Mortalidade Padronizada por Suicídio no período de 2000-2003: A-GINI, B- IDHM; C- Taxa de Vulneráveis a pobreza; D- Desocupação; E- População rural; FEnvelhecimento; G- Analfabetismo.

quando comparadas aos valores brutos e padronizados. Os resultados de uma pesquisa realizada por Macente e Zandonade ${ }^{30}$ para municípios do Espírito Santo corroboraram com essa análise aportada no presente estudo, mostrando variação na distribuição espacial a partir da utilização de outras técnicas estatísticas.

Com relação à análise de tendência, esse estudo mostrou que não houve tendência de aumento das taxas de suicídio ao longo dos anos para ambos os sexos. Porém, ao se analisar o sexo masculino, percebeu-se uma tendência estatisticamente significativa $(\mathrm{p}=0,03)$ de aumento da mortalidade por essa causa nos primeiros anos da série histórica estudada. Os resultados de um estudo realizado em um município no interior de Minas Gerais corroboraram com esses dados, mostrando um padrão de tendência de aumento significativo para os homens (APVP $=7,2$ a $8,8 \%)^{31}$.

Alguns autores ${ }^{32}$, ao analisarem a tendência para as taxas de suicídio no Brasil, constataram uma heterogeneidade espaço-temporal. Foi observado ainda que entre 1980 e 2005 as taxas au- mentaram em ambos os sexos, sobretudo com o avanço da idade, e que os maiores coeficientes de mortalidade foram os de São Paulo, Sul e Centro -Oeste do país, independente de idade e sexo.

Em outros estudos ${ }^{33}$, ao analisarem a tendência das taxas de suicídio de 1996 a 2009 para o estado de São Paulo, foi verificado para os homens acima de 65 anos uma redução significativa de $2,3 \%$ por ano. Para as mulheres, porém, as taxas foram estáveis durante todo o período. Neves e Garcia $^{34}$ observaram tendência crescente entre homens nas regiões Nordeste e Sul $(3,08 \%$ e 0,88\% respectivamente).

Em países como a Espanha, majoram investigações sobre tendências de mortalidade por suicídio utilizando a regressão joinpoint. Entre os anos 2000 e 2011, um estudo mostrou que as taxas globais de suicídio apresentaram uma tendência decrescente significativa $(1,93 \%$ em homens e $2,19 \%$ em mulheres), enquanto que para os anos 2012 a 2015 foi observado um aumento significativo $(3,65 \%$ em homens e $7,60 \% \mathrm{em} \mathrm{mu}-$ lheres) ${ }^{35}$. 


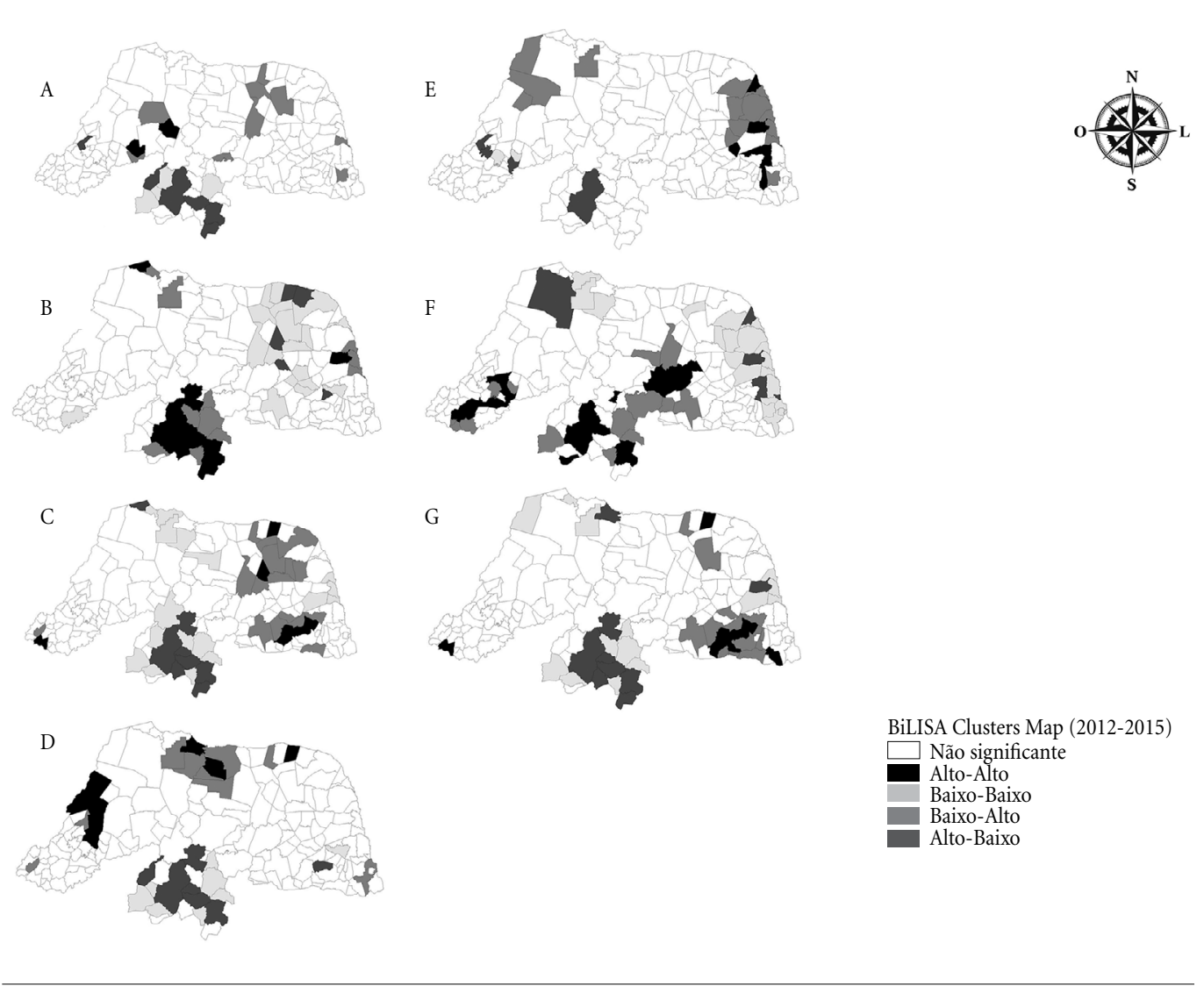

Figura 5. Moran Bivariado LISA entre as Taxas de Mortalidade Padronizada por Suicídio no período de 2012 a 2015: A-GINI, B- IDHM; C- Taxa de Vulneráveis a pobreza; D- Desocupação; E- População rural; FEnvelhecimento; G- Analfabetismo.

Desse modo, a mortalidade por suicídio está desigualmente distribuída em todo o RN, e apresentou tendência de aumento ao longo de um período da série histórica para o sexo masculino. Por outro lado, os óbitos por suicídio não estão relacionados às piores condições de vida, uma vez que indicadores socioeconômicos como Índice de Desenvolvimento Humano e Envelhecimento estiveram associados às altas taxas de mortalidade, sobretudo em regiões bem desenvolvidas, como a região do Seridó.

Os achados fortalecem a necessidade de se buscarem explicações para as diferenças dos índices de suicídio em seu contexto local, apontando a importância da organização da vigilância epidemiológica e a pesquisa focada para os municípios de maior ocorrência, de forma a favorecer uma compreensão desse agravo na saúde pública, bem como melhorar as possibilidades de prevenção.

A situação da mortalidade por suicídio no RN pode ser reflexo da ausência de programas de prevenção e despreparo dos profissionais de saúde e sociedade em geral durante a abordagem ao paciente vítima de tentativa de suicídio. Desse modo, a implantação de políticas públicas de saúde direcionadas às populações mais afetadas; a elaboração de programas de prevenção e a capacitação dos profissionais em todos os níveis de atenção à saúde e sociedade em geral podem reduzir a mortalidade por esta causa no estado.

A limitação desse estudo está pautada na utilização de dados secundários acerca da mortalidade, que está sujeita à subregistro, embora se reconheça que o Sistema de Informação sobre Mortalidade no Brasil obteve um ganho significativo de qualidade. Atrelado a isso, acredita-se que o ganho na qualidade dos dados pode estar relacionado à tendência de aumento nos primeiros anos da série histórica. Ademais, esse estudo amplia o olhar para a importância da qualificação, manejo efetivo dos dados provenientes do SIM e (re) estruturação da atenção em saúde mental no $\mathrm{RN}$, considerando as peculiaridades dos grupos vulneráveis ao suicídio. 


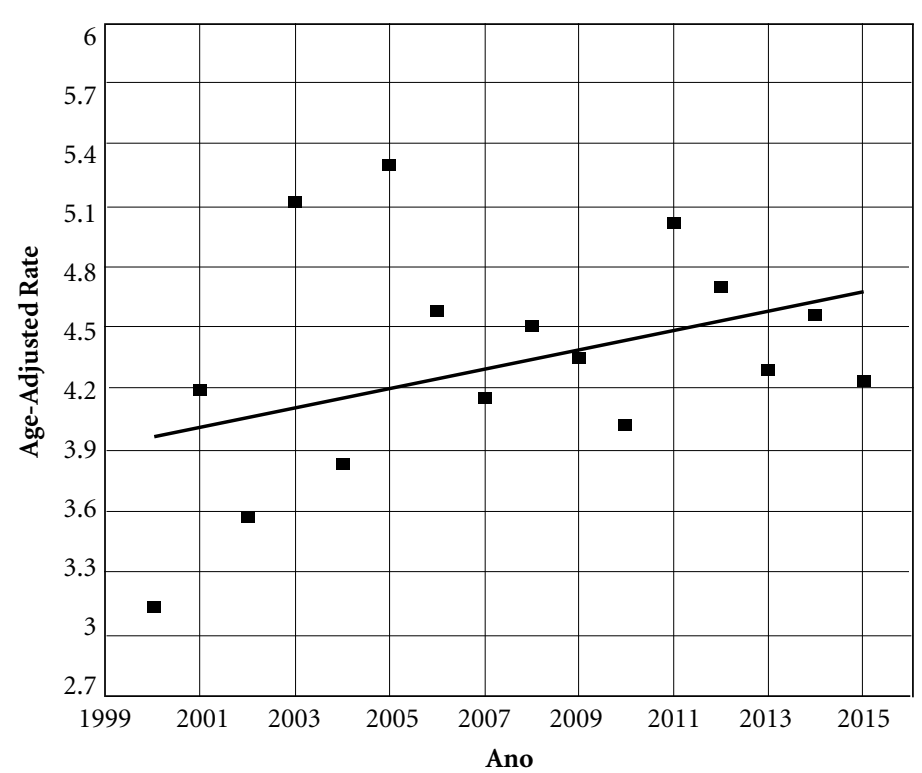

Observed

2000.0-2015 APC $=1.09$

Gráfico 1.Tendência de mortalidade por suicídio no RN: All: 0 Joinpoints.

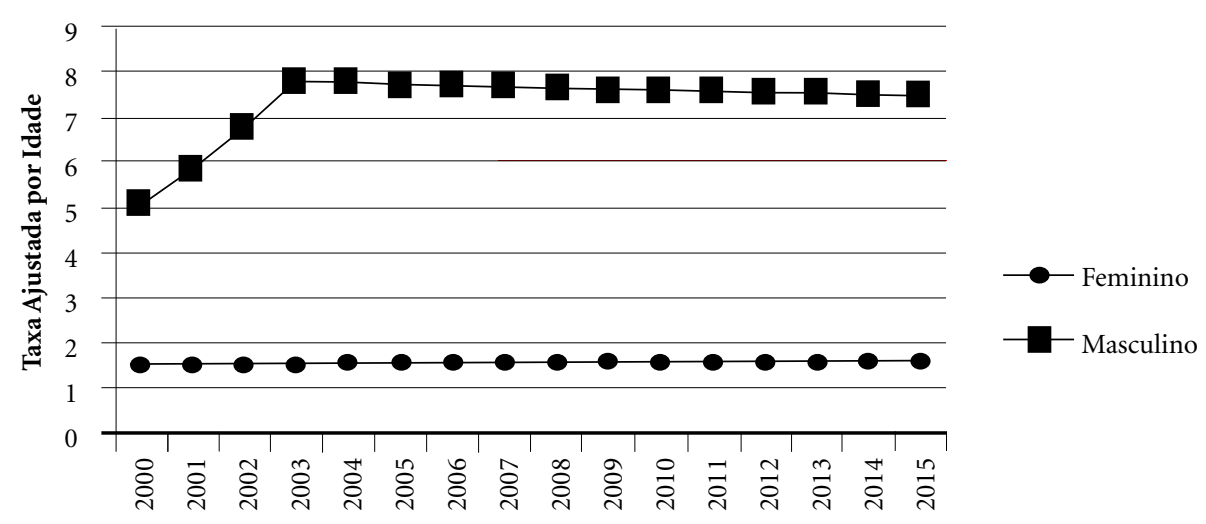

Ano

Gráfico 2.Tendência de mortalidade por suicídio no RN (2000-2015) segundo sexo.

Tabela 1. Tendência temporal da mortalidade por suicídio no RN: número de óbitos, Annual Percentage Change (APC), Intervalo de Confiança, RN, 2017.

\begin{tabular}{lcrcccc}
\hline \multicolumn{1}{c}{ RN } & No de óbitos & APC1 (IC 95\%) & p-value & Joinpoint & APC2 (IC 95\%) & p-value \\
\hline Ambos os sexos & 2266 & $1,1(0,6-1,6)$ & 0,25 & & & \\
Feminino & 430 & $0,4(-0,9-1,7)$ & 0,69 & & & \\
Masculino & 1836 & $\mathbf{1 5 , 3 ^ { * }}(\mathbf{9 , 2}-\mathbf{2 1 , 8})$ & 0,03 & $\mathbf{2 0 0 3}$ & $-0,4(-1,0-0,3)$ & 0,01 \\
\hline
\end{tabular}




\section{Colaboradores}

EGO Santos, IR Barbosa e AKS Severo participaram das fases dessa publicação em uma ou mais etapas a seguir: participação substancial na concepção ou confecção do manuscrito ou da coleta, análise ou interpretação dos dados; elaboração do trabalho ou realização de revisão crítica do conteúdo intelectual; aprovação da versão submetida.

\section{Referências}

1. Associação Brasileira de Psiquiatria. Suicídio: Informando para prevenir. Brasília: Conselho Federal de Medicina; 2014.

2. Brasil. Ministério da Saúde (MS). Suicídio. Saber, agir e prevenir. Brasília: MS; 2017. [Boletim epidemiológico]

3. Romeder JM, Mcwhinnie JR. Años de vida potencial perdidos entre las edades de 1 y 70 años: un indicator de mortalidad prematura para la planificatión de la salud. In: Buck C, Llopls A, Najera E, Terris M, organizadores. El Desafio de la Epidemiologia. Washington: OPAS; 1988.

4. Pontes RJS, Ramos-Júnior AN, Kerr LRS, Bosi MLM. Transição demográfica e epidemiológica. In: Medronho RA, Bloch KV, Luiz RR, Werneck GL, organizadores. Epidemiologia. 2a ed. São Paulo: Atheneu; 2009.

5. World Health Organization (WHO). Preventing suicide: a global imperative. Geneva: WHO; 2014.

6. Brasil. Departamento de Informática do SUS. Sistema de Informação sobre Mortalidade. [acessado $2017 \mathrm{Mar}$ 5]Disponível em: http://datasus.saude.gov.br/

7. Botega N, Bertolote JM, Hetem LA, Bessa MA. Prevenção do suicídio. Debates: psiquiatria hoje 2010; 2(1):10-20.

8. Medronho RA. Estudos ecológicos. In: Medronho RA, Bloch KV, Luiz RR, Werneck GL, organizadores. Epidemiologia. São Paulo: Editora Atheneu; 2009.

9. Medeiros WR. Mortalidade em idosos longevos e "mais jovens" no Brasil [tese]. Natal: Universidade Federal do Rio Grande do Norte; 2015.

10. Mello-Jorge MHP, Laurenti R, Gotlieb SLD. Análise da qualidade das estatísticas vitais brasileiras: a experiência de implantação do SIM e do SINASC. Cien Saude Colet [revista na internet] 2007 [acessado $2017 \mathrm{Nov}$ 20]; 12(3):643-654. Disponível em: http://www.scielo. br/pdf/\%0D/csc/v12n3/14.pdf

11. Felix JD, Zadonade EL, Amorim MHC, Castro DS Avaliação da completude das variáveis epidemiológicas do Sistema de Informação sobre Mortalidade em mulheres com óbitos por câncer de mama na Região Sudeste - Brasil (1998 a 2007). Cien Saude Colet [revista na internet] 2012 [acessado 2017 Nov 20]; 17(4):945-953. Disponível em: http://www.redalyc. org/html/630/63023349016/

12. Anais do XI Encontro Nacional de Estudos Populacionais, 1998, Caxambu-MG. Caxambu: ABEP, 1998.

13. Instituto de Pesquisa Econômica Aplicada (IPEA). Atlas da violência. Rio de Janeiro: IPEA; 2017.

14. Instituto Brasileiro de Geografia e Estatística (IBGE). Projeção da população do Brasil e das Unidades da Federação. Rio de Janeiro: IBGE; 2017.

15. Instituto Nacional de Pesquisas Espaciais (INPE). Análise exploratória do consumo per capita de água residencial e renda per capita, em um setor de abastecimento de água. INPE; 2013.

16. Bezerra Junior JGO, Silva NM. Caracterização geoambiental da microrregião do seridó oriental do Rio Grande do Norte. Rev Holos 2007; 23(2):78-91.

17. Dutra E. Epidemiologia do suicídio no RN: 1985 a 1996. Anais da 50a Reunião da Sociedade Brasileira para o Progresso da Ciência, Natal-RN. 1997. 
18. Fraga WS. Determinantes socioeconômicos do suicídio no Brasil e Rio Grande do Sul no século XXI [dissertação]. São Leopoldo: Universidade do Vale do Rio dos Sinos; 2014.

19. Fountoulakis KN, Chatzikosta I, Pastiadis K, Zanis P, Kawohl W, Kerkhof AJFM, Navickas A, Höschl C, Lecic-Tosevski D, Sorel E, Rancans E, Palova E, Juckel G, Isacsson G, Jagodic HK, Botezat-Antonescu I, Rybakowski J, Azorin JM, Cookson J, Waddington J, Pregelj P, Demyttenaere K, Hranov LG, Stevovic LI, Pezawas L, Adida M, Figuera ML, Jakovljević M, Vichi M, Perugi G, Andreassen OA, Vukovic O, Mavrogiorgou P, Varnik P, Dome P, Winkler P, Salokangas RKR, From T, Danileviciute V, Gonda X, Rihmer Z, Forsman J, Grady A, Hyphantis T, Dieset I, Soendergaard S, Pompili M, Bech P. Relationship of suicide rates with climate and economic variables in Europe during 2000-2012. Ann Gen Psychiatry [revista na internet]. 2016 [acessado 2017 Nov 1]; 15:19. Disponível em: https://www.ncbi.nlm.nih.gov/pmc/articles/PMC4977895/

20. Santos EGO, Barbosa IR. Conglomerados espaciais da mortalidade por suicídio no nordeste do Brasil e sua relação com indicadores socioeconômicos. Cad saúde colet [revista na internet] 2017 [acessado $2017 \mathrm{Nov}$ 1]; 25(3):371-378. Disponível em: http://www.scielo. br/scielo.php?pid=S1414-462X2017000300371\&scri $\mathrm{pt}=$ sci_abstract\&tlng $=\mathrm{pt}$

21. Hamermesh D. Na economic theory of suicide. Journal of Political Economy 1974; 82(1).

22. Durkheim E. O suicídio. Lisboa: Presença; 2003

23. Machado DB, Santos DN. Suicídio no Brasil, de 2000 a 2012. J Bras de Psiquiatr [revista na internet]. 2015 [acessado 2017 Nov 10]; 64(1). Disponível em: http:// www.scielo.br/pdf/jbpsiq/v64n1/0047-2085-jbpsiq-64-1-0045.pdf

24. Chen YY, Pei-Chen T, Pao-Huan C, Chun-Chieh $\mathrm{F}$, Galen $\mathrm{CH}$, Andrew TA. Effect of media reporting of the suicide of a singer in Taiwan: the case of lvy Li. Sco Psychiatry Epidemiol [revista na internet] 2009 [acessado 2017 Nov 2]; 45(3):363-369. Disponível em: https://link.springer.com/article/10.100 7\%2Fs00127-009-0075-8

25. Gonçalves LRC, Gonçalves E, Oliveira-Júnior LB. Determinantes espaciais e socioeconômicos do suicídio no Brasil: uma abordagem regional. Nova econ [revista na internet] 2011 [acessado 2017 Nov 1]; 21(2). Disponível em: http://www.scielo.br/scielo.php?pid =S0103-63512011000200005\&script $=$ sci_arttext\&tl$\mathrm{ng}=\mathrm{es}$.

26. Rehkopf DH, Buka SL. The association between suicide and the socio-economic characteristics of geographical áreas: a systematic review. Psychol Med [revista na internet]. 2006 [acessado 2018 Mar 15]; 36(2):145-157. Disponível em: https://www.ncbi.nlm. nih.gov/pubmed/16420711.

27. Vijayakumar L, Nagaraj K, Pirkis J, Whiteford H. Suicide in developing countries: frequency, distribuition, and association whit socioeconomic indicators. Crisis [revista na internet]. 2005 [acessado em $2018 \mathrm{mar}$ 15];26(3):104-111. Disponível em: https://econtent. hogrefe.com/doi/abs/10.1027/0227-5910.26.3.104.
28. Gonçalves LRC, Gonçalves E, Oliveira Júnior LB. Determinantes espaciais e socioeconômicos do suicídio no Brasil: uma abordagem regional. Nova econ [revista na internet]. 2011 [acessado em 2017 Nov 15]; 21(2). Disponível em: http://www.scielo.br/scielo.php ?pid=S0103-63512011000200005\&script $=$ sci_arttext\&tlng=es

29. Campos MB, Barbieri AF. Considerações teóricas sobre as migrações de idosos. Rev Bras Est Pop [revista na internet] 2013 [acessado 2017 Nov 14]; 30:69-84. Disponível em: http://www.scielo.br/pdf/rbepop/ v30s0/05.pdf.

30. Macente LB, Zandonade E. Spatial distribution of suicide incidence rates in municipalities in the state of Espírito Santos (Brazil), 2003-2007: spatial analysis to identify risk areas. Rev Bras Psiquiatr [revista na internet] 2012 [acessado 2017 Nov 12]; 34 (3). Disponível em: http://www.scielo.br/scielo.php?script=sci_arttext\&pid=S1516-44462012000300005\&lng=en\&n$\mathrm{rm}=\mathrm{iso} \& \operatorname{tng}=\mathrm{en}$.

31. Bando DH, Souza FD, Silva HLR, Bressan VR, Silva AS. Tendência das taxas de suicídio no município de Alfenas (MG), 1996 a 2012. In: $4^{a}$ Jornada Científica da Geografia UNIFAL-MG, 2016; Alfenas-MG. p. 465570.

32. Brzozowski FS, Soares GB, Benedet J, Boing AF, Peres MA. Suicide time trends in Brazil from 1980 to 2005. Cad Saude Publica [revista na internet] 2010 [acessado 2017 Nov 16]; 26(7). Disponível em: http://www.scielo.br/scielo.php?script=sci_arttext\&pid=S0102-311X2010000700008

33. Bando DH, Brunoni AR, Fernandes TG, Benseñor IM, Lotufo PA. Suicide rates and trends in São Paulo, Brazil, according to gender, age and demographic aspects: a joinpoint regression analysis. Rev Bras Psiquiatr [revista na internet] 2012 [acessado 2017 Nov 5]; 34:286-293. Disponível em: http://www.scielo.br/pdf/ $\mathrm{rbp} / \mathrm{v} 34 \mathrm{n} 3 / \mathrm{v} 34 \mathrm{n} 3 \mathrm{a} 08 . \mathrm{pdf}$.

34. Neves ACM, Garcia LP. Youth mortalitu in Brazil: profile and trends in the period 2000-2012. Epidemiol. Serv. Saúde [revista na internet] 2015 [acessado 2017 Nov 5]; 24(4) Disponível em: http://scielo.iec.pa.gov. br/scielo.php?pid=S1679-49742015000400002\&scri $\mathrm{pt}=$ sci_arttext\&tlng $=$ en

35. Delfrade J, Sayon-Orea C, Teijeira-Álvarez R, Floristán-Floristán Y, Moreno-Iribas C. Divergent trends in suicide mortality in Navarra and Spain: 2000-2015. Rev Esp Salud Publica [revista na internet] 2017 [acessado 2017 Nov 12]; 3(91). Disponível em: https:// www.ncbi.nlm.nih.gov/pubmed/28463956
Artigo apresentado em 02/12/2017

Aprovado em 01/06/2018

Versão final apresentada em 03/06/2018 
